\title{
Agentes comunitários de saúde e agentes de combate a endemias: Desafios para controle do Aedes aegypti
}

\author{
Fabíola Olinda de Souza Mesquita ; Alaine Santos Parente ${ }^{2}$; Glória Maria Pinto Coelho ${ }^{3}$.
}

\begin{abstract}
Resumo: O controle das doenças transmitidas pelo vetor Aedes aegypti está relacionado a um conjunto de ações ligadas a atenção primária a saúde, vigilância em saúde, educação em saúde e participação social. Considerando a importância da realização de ações integradas o objetivo desse estudo foi identificar como ocorre a articulação dos Agentes Comunitários de Saúde (ACS) com os Agentes de Combate a Endemias (ACE) no cotidiano das ações de prevenção e controle do Aedes aegypti. Foi realizado revisão de literatura através da análise de artigos publicados no período de 2011 a 2016 na base de dados Scientific Electronic Library Online (Scielo) e Biblioteca Virtual em Saúde (BVS). A partir da análise observou-se que as ações de controle ao vetor exigem dos profissionais, um trabalho integrado, multiprofissional. As ações extrapolam o setor saúde sendo indispensável fomentar políticas intersetoriais, ampliar a educação permanente dos profissionais e estimular a participação ativa da sociedade.
\end{abstract}

Palavras-chaves: Aedes aegypti. Atenção Primária à Saúde. Vigilância em Saúde.

\section{Community health agents and agents to combat endemic diseases: Challenges to control Aedes aegypti}

\begin{abstract}
The control of diseases transmitted by the Aedes aegypti vector is linked to a set of actions related to primary health care, health surveillance, health education and social participation. Considering the importance of carrying out integrated actions, the goal of this study was to identify how the articulation of the Community Health Agents (ACS) with the Agents of Endemic Diseases (ACE) occurs in the daily activities of prevention and control of Aedes aegypti. The review of the literature had carried out through the analysis of articles published in the period from 2011 to 2016 in the Scientific Electronic Library Online (Scielo) and Virtual Health Library (BVS) database. From the analysis it was observed that the control actions to the vector require the professionals, an integrated, multiprofessional work. The actions extrapolate the health sector and it is essential to foster intersectoral policies, increasing the permanent education of professionals and stimulating the active participation of society.
\end{abstract}

Keywords: Aedes aegypti. Primary Health Care. Health Surveillance.

\footnotetext{
${ }^{1}$ Graduada em Odontologia pela Universidade Federal de Pernambuco; Especialização em Gestão em Saúde pela Universidade Federal do Vale do São Francisco (UNIVASF). E-mail: fabiolaolinda@yahoo.com.br.

${ }^{2}$ Graduada em Fisioterapia pela Faculdade Santa Maria- Cajazeiras- PB; Especialização em Gestão em Saúde pela Universidade Federal do Vale do São Francisco (UNIVASF). E-mail: alainefisio@hotmail.com.

${ }^{3}$ Graduada em Enfermagem pela Universidade Católica de Salvador; Mestrado em Ciências pela Universidade Federal de São Paulo. E-mail: gloriapintocoelho@gmail.com.
} 


\section{Introdução}

No início do século XX, o mosquito Aedes aegypti foi identificado como transmis da febre amarela urbana e em 1955, ocorre a sua erradicação no Brasil, no entanto, houve a reintrodução por meio de deslocamentos humanos marítimos ou terrestres (BRAGA; VALLE, 2007; PERES, A.C, 2016).

Na segunda metade do século XX, a partir de 1981, ocorreu na cidade de Boa Vista/RR, o primeiro registro epidêmico da arbovirose dengue, estendendo-se posteriormente, para outros estados. Na década de 90 , a dispersão do vetor atingiu grande parte do país ocasionando vários surtos nos grandes centros urbanos das regiões Sudeste e Nordeste (SANTOS, 2012).

Em 2014, casos de Chikungunya foram confirmados em indivíduos oriundos de países da América Central e os primeiros casos autóctones foram identificados no Amapá. Em 2015, o Ministério da Saúde confirmou casos do Zika vírus no Brasil que rapidamente se espalharam em vários nos estados do País (CHAVES et al., 2015).

O protagonismo da comunidade na eliminação do mosquito é essencial, pois estudos apontam que cerca de $90 \%$ dos criadouros estão no interior dos domicílios (GOMES et al.,2015). As instituições governamentais têm buscado organizar ações integradas de saúde, educação, comunicação e mobilização social, na Atenção Primária a Saúde, em especial a Estratégia Saúde da Família (ESF). Há fatores que intervêm na aderência da população às práticas preventivas que tem relação direta com a falta de comunicação entre o serviço de saúde e a comunidade. Se faz necessário prover ambientes que estimulem a discussão nas comunidades sobre as questões sanitárias relevantes e de forma permanente (SILVA et al., 2011).

Para combater o Aedes é imperativo repensar a configuração de intervenções no meio ambiente, pois sem a implantação de sistemas públicos de abastecimento de água para consumo doméstico de forma regular, esgotamento sanitário, o correto destino dos resíduos sólidos urbanos e sem redução da violência em muitas comunidades urbanas não há como reduzir os índices de infestação dos mosquitos (PERES, 2016). 
As doenças causadas por patógenos veiculados por vetores estão diretamente relacionadas à precariedade de condições sociais e sanitárias. $\mathrm{O}$ atual programa de controle do mosquito não abrange políticas públicas voltadas para a solução da grave desigualdade relativa a tais condições, tornando ineficientes as medidas de promoção, de vigilância e de educação em saúde (CESARINO et al., 2014).

As ações de educação em saúde exigem interação de conhecimentos, a partir de uma relação pautada em uma relação horizontal, considerando as necessidades dos participantes transformando-os em sujeitos construtores de suas vidas em uma conjuntura sócio cultural, permeado por expectativas, valores, crenças e hábitos (SILVA et al., 2011).

As políticas públicas setoriais estão estruturadas para funcionarem isoladamente, em função do grau de especialização e profissionalização de cada setor. A educação permanente na perspectiva da intersetorialidade baseia-se na articulação das ações de gestão sobre problemas reais, elencados pelos sujeitos que vivem nesses territórios, e que são transformadas em demandas políticas. Esse é o papel central que a prática da vigilância em saúde deve desempenhar na atenção primária à saúde (CESARINO et al., 2014).

A Portaria Ministerial GM N ${ }^{0} 2121 / 2015$ acrescenta como atribuições dos Agentes Comunitários de Saúde (ACS) executar em conjunto com Agentes de Combate a Endemias (ACE), em situação de surtos e epidemia, ações de controle de doenças, utilizando as medidas de controle adequadas, manejo ambiental e outras ações de manejo integrado de vetores, de acordo com decisão da gestão municipal (BRASIL,2015).

Considerando a importância da realização de ações integradas, essa pesquisa tem como objetivo identificar como ocorre a articulação do ACS com o ACE no cotidiano das ações de prevenção e controle do A. aegypti na Atenção Primária à Saúde além das estratégias de enfrentamento adotadas nos municípios de todo território nacional.

Os resultados poderão ser utilizados pelos gestores a fim de que possam comparar as estratégias já implantadas e executadas nos municípios de forma a potencializar ações de promoção e controle do mosquito além de permitir a implantação de políticas com a participação da comunidade e das instituições sejam públicas ou privadas. 


\section{Metodologia}

O estudo é uma revisão de literatura com base em pesquisa sistemática de estudos publicados na base de dados Scientific Eletronic Library Online - Scielo e, Biblioteca Virtual em Saúde- BVS. Os delimitadores utilizados foram representados pelos conectores "e" significando uma combinação restritiva e "ou" uma combinação aditiva. A utilização desses conectores possibilitou maior número de combinações com os descritores: Aedes aegyti, atenção primária, vigilância em saúde e promoção em saúde contribuindo para melhor refinamento do objeto de estudo. Vale ressaltar que o quantitativo de artigos pesquisados foram 33, no entanto, apenas 20 artigos compuseram o referido estudo.

A escolha dos artigos foi relacionada com artigos que versavam sobre o protagonismo dos agentes comunitários de saúde e agente de combate as endemias e a inter-relação dessas categorias profissionais as estratégias de enfrentamento utilizadas para o controle ao Aedes aegypti em diversas localidades do território nacional.

Entretanto, excluíram-se artigos escritos em língua estrangeira, artigos publicados há mais de seis anos, artigos que só estavam disponíveis os resumos, e que a ideia principal versava sobre o ciclo de desenvolvimento do Aedes aegypti, sorotipos do vírus, modo de aplicação de larvicidas, classificação de risco, manejo clínico e tratamento, estudos epidemiológicos realizados a partir de banco de dados do Sistema de Informação de Agravos e Notificação (SINAN).

Para análise dos dados, considerou-se a normatização do Programa Nacional de Controle da Dengue (PNCD) do Ministério da Saúde que estabelece os critérios que devem nortear os municípios para o controle do vetor (BRASIL,2002) e compará-los aos dados obtidos com o referencial teórico fundamentador dessa temática.

Outrossim, avaliar o impacto dos fatores como: precárias condições sanitárias dos centros urbanos, abastecimento intermitente de água dentre outros fatores, que propiciam o desenvolvimento das diversas formas evolutivas do vetor foi o parâmetro basilar para observar o que tem sido realizado nos municípios para o combate e prevenção do Aedes aegypti conforme os artigos científicos no período de 2011 a 2016.

Com base nesses aspectos os textos foram categorizados nas seguintes dimensões: 
- Agentes Comunitários de Saúde (ACS) e os Agentes de Combate as Endemias (ACE): combate ao vetor e integração/articulação entre categorias profissionais.

- Agentes Comunitários de Saúde (ACS) e os Agentes de Combate as Endemias (ACE): ações integradas de educação em saúde, comunicação e mobilização social.

- Agentes Comunitários de Saúde (ACS) e os Agentes de Combate as Endemias (ACE): papel do saneamento ambiental.

\section{Resultados e Discussão}

Os estudos elencados focaram o Aedes aegypti em relação às medidas de combate ao vetor, qualidade das condições socioambientais, a educação permanente, a mobilização social e intersetorialidade.

\section{Agentes Comunitários de Saúde (ACS) e os Agentes de Combate as Endemias (ACE): combate ao vetor e integração/articulação entre as categorias profissionais.}

Quanto ao processo de inserção dos ACE nas Unidades Básicas de Saúde (UBS) em pesquisa realizada em São José do Rio Preto- SP, com a finalidade de melhorar a eficácia do programa de controle da dengue observaram que as atividades de promoção a saúde, de prevenção das doenças e manejo ambiental centrada num conceito mais amplo de saúde junto aos ACE permitiu compartilhar as experiências e ampliou o olhar do agente de combate as endemias e estes passaram a se sentir valorizados pela comunidade e pela Equipe de Saúde da Família (ESF) além de desempenharem suas atividades com maior motivação (CESARINO et al., 2014).

Por outro lado, Libanio; Favoreto; Pinheiro, (2014) realizaram um estudo a fim de analisar a incorporação das ações de Vigilância Ambiental pelos ACS, no período de 2006 a 2013, no município de Piraí-RJ, os quais passaram a identificar os criadouros de larvas, 
aplicar biolarvicidas, a colocação de telas em reservatórios de água, entre outros. Como resultado houve melhoria dos dados da vigilância da dengue com cobertura em visitas em 90\% dos imóveis existentes, melhor identificação de áreas e domicílios de risco e execução de atividades educativas relacionadas aos territórios.

De outra forma, o vínculo dos ACS com a comunidade, em especial, os da zona rural, trouxe desafios como aplicação de notificações e intervenções legais sobre o ambiente, pois havia dificuldade da população em reconhecê-los como agentes que executam a ação de vigilância de dengue. Os ACS reconhecem a necessidade de momento de integração com a vigilância a fim de dirimir conflitos, avaliar e monitorar o trabalho executado e buscarem estratégias que propiciem a integração através do processo de educação permanente (LIBANIO; FAVORETO; PINHEIRO, 2014; OLIVEIRA et al, 2012).

Em oposição, no estudo de Santos et al., (2015) verificou-se que não há integração entre a ESF e o Programa de Dengue, tanto no que se refere às atividades de campo do ACS, quanto pela equipe de saúde, já que a dengue não é uma doença de interesse, incorporada na rotina de suas atividades. A falta de aproximação entre o programa da Dengue e o de Saúde da Família é reconhecida como uma dificuldade nos resultados do programa. No entanto, o PNCD (2002) destaca no componente Integração com Atenção Básica que o objetivo principal é consolidar a inserção do PACS e da Estratégia em Saúde da Família nas ações de prevenção e controle da dengue, visando, principalmente, promover mudanças de hábitos na comunidade para manter o ambiente doméstico livre do Aedes aegypti. Além de ações educativas os ACS contribuirão para aumentar a sensibilidade do sistema de vigilância por meio de notificação imediata da ocorrência de casos. Enfatiza a necessidade de unificação das áreas geográficas de trabalho dos ACS e dos ACE possibilitando uma ação mais oportuna quando ocorrer à detecção de focos do mosquito (BRASIL, 2002).

É importante salientar que a identificação dos fatores que dificultam ou potencializam o processo de incorporação dos agentes de endemias nas atividades de prevenção e controle da dengue na Estratégia Saúde da Família facilita o mecanismo de cooperação e coordenação entre profissionais e serviços de saúde (PESSOA et al., 2016).

No estudo de Pessoa et al., (2016) apontaram que os ACS e ACE identificaram necessidade de realizar uma capacitação funcional entre esses profissionais e todos os 
profissionais de saúde incluindo os supervisores e gestores com sensibilização dos envolvidos com a finalidade de favorecer a realização de atividades integradas. Destacam que ao detalhar as funções dos profissionais, permite que o trabalho dos agentes seja maximizado, atuando em parceria (ACE/ACS) no controle da dengue.

\section{Agentes Comunitários de Saúde (ACS) e os Agentes de Combate as Endemias (ACE): ações integradas de educação em saúde, comunicação e mobilização social.}

Na pesquisa de Libanio; Favoreto; Pinheiro, (2014) salientaram dificuldades dos ACS em integrar as ações de educação/promoção de saúde com a vigilância da dengue. A perspectiva fiscalizadora das ações de controle ao vetor impossibilitava que a comunidade percebesse a vigilância da dengue também numa perspectiva de educação e promoção em saúde.

Outrossim, discorreram que apesar do estreito vínculo dos ACS com a comunidade não houve referência espontânea a mobilização comunitária nem foram abordadas questões referentes aos determinantes sociais e culturais que interferem no comportamento dos moradores nem tampouco ações que envolvessem outras instituições locais. Os ACS não conseguiram entender o papel integrado da Vigilância ambiental em saúde com mobilização comunitária e intersetorialidade (LIBANIO; FAVORETO; PINHEIRO, 2014).

Os ACS e ACE sentem necessidade de capacitação para o trabalho na perspectiva da integralidade do atendimento, se fazendo necessário a criação de um espaço de discussão no qual o saber e fazer, num processo de educação permanente, seja incorporado à prática concreta dos serviços de saúde (LIBANIO; FAVORETO; PINHEIRO, 2014 ; CESARINO et al, 2014).

A educação permanente deve ser indispensável no que tange a qualificação das práticas de cuidado, gestão e participação popular. Nesse sentido, a educação permanente é educação no trabalho, pelo trabalho e para o trabalho nos díspares espaços de assistência, cuja finalidade é ofertar saúde de qualidade a saúde da população (CESARINO et al, 2014).

A eficácia de estratégias utilizadas para o combate ao mosquito Aedes aegypti com foco no controle do vetor está comprovada por diversos estudos, mas é imperativo reconhecer 
que as ações voltadas à mudança de condutas da população são fundamentais e que sem elas o controle sempre será um problema de saúde pública (REIS, ANDRADE, CUNHA, 2013).

O PNCD salienta que o principal objetivo do componente ações integradas de educação em saúde, comunicação e mobilização social é estimular o desenvolvimento de ações educativas para a mudança de comportamento e adoção de práticas de manutenção do ambiente domiciliar preservado da infestação por Aedes aegypti, observadas a sazonalidade da doença e as realidades locais quanto aos principais criadouros (BRASIL,2002).

A comunicação social terá como objetivo divulgar e informar sobre ações de educação em saúde e mobilização social para mudança de comportamento e de hábitos da população, buscando evitar a presença e a reprodução do Aedes aegypti nos domicílios por meio da utilização dos recursos disponíveis na mídia.

Um alto nível de conhecimento sobre a dengue não se reflete em prática preventiva adequada e eficaz para redução da ocorrência da doença. A comunicação deve gerar referenciais para a ação e para mudança de comportamentos nos indivíduos de acordo a sua trajetória pessoal e social (REIS; ANDRADE; CUNHA, 2013; SANTOS et al., 2011).

Torna-se imprescindível que tanto os sujeitos receptores sejam comunicantes quanto a comunicação traduza para o sujeito receptor conteúdos significativos comuns. Para tal finalidade é imperativo o processo de educação permanente como estratégia para manter e qualificar as ações integradas de vigilância ao Aedes aegypti (LIBANIO; FAVORETO; PINHEIRO, 2014; CAZOLA et al., 2014).

Canais privilegiados de compartilhamento de saberes, é fundamental para as práticas pedagógicas, pois impede reproduzir modelos de transmissão linear das informações, assemelhando-se às pedagogias tradicionais. No campo da saúde, essa atitude manifesta-se no costume demasiadamente prescritivo e na distância de resultados de pesquisas científicas das práticas de políticas públicas de prevenção (OLIVEIRA, 2012).

A atividade educativa voltada para a comunidade é um potencial mobilizador popular no controle da dengue, no entanto, estudos mostram que a realização dessas atividades sem um bom planejamento compromete sua execução e não garante ações efetivas que consigam reduzir a prevalência da doença (SILVA et al., 2011). 
Com base no PNCD (2002) o componente sustentação político-social tem como objetivo sensibilizar e mobilizar os setores públicos com o intuito de garantir o aporte financeiro e a articulação intersetorial necessários à implantação e execução do programa (BRASIL, 2002).

A intersetorialidade tem como alicerce o conceito de território enquanto espaço geográfico e político social, no qual a identificação dos macroproblemas de saúde deve gerar um conjunto de operações intersetoriais com o objetivo de resolvê-los, considerando a disponibilidade real de recursos existentes nesse território (CESARINO et al.,2014).

Na pesquisa, Cesarino et al (2014), observou-se que os ACE e ACS não estavam preparados para lidar com questões relacionadas a problemas da coletividade e com a intersetorialidade. Nesse estudo, o supervisor da equipe recebia as demandas dos ACE e só existiam fluxos dentro da própria Secretaria Municipal de Saúde (SMS), um fluxo intrasetorial. As questões que demandavam ações intersetoriais eram encaminhadas para a coordenação do programa. Os ACS quando realizavam as visitas domiciliares e se comprometiam em solucionar determinado problema, tinham suas demandas postergadas em virtude da morosidade na resolução das mesmas.

A intersetorialidade é uma ferramenta de políticas públicas e de promoção à saúde e exige maior articulação com a população com o objetivo de fomentar sua participação nos processos decisórios quanto a saúde individual e da coletividade, tornando suas relações mais horizontalizadas (LIMA; VILASBOAS, 2011).

\section{Agentes Comunitários de Saúde (ACS) e os Agentes de Combate as Endemias (ACE): papel do saneamento ambiental.}

O PNCD (2002) afirma a necessidade de fomentar ações de saneamento ambiental para um efetivo controle do Aedes aegypti buscando garantir fornecimento contínuo de água, a coleta e a destinação adequada dos resíduos sólidos e a correta armazenagem de água no domicílio, onde isso for imprescindível (BRASIL,2002).

Em consequência do não estímulo ao estabelecido no PNCD a disseminação do mosquito tem sido favorecida pelas precárias condições sanitárias dos centros urbanos, 
abastecimento intermitente de água dentre outros fatores que propiciam o desenvolvimento das diversas formas evolutivas do vetor (SANTOS, 2012).

O PNCD aponta como ações: fomentar a limpeza urbana e a coleta regular de lixo a qual deve ser realizada de forma sistemática pelos municípios, desenvolver modelos de reservatórios para armazenamento de água potável em domicílios. Além disso, propõe alterações nas normas para a fabricação de caixas de água adaptando-as contra a infestação pelo aedes aegypti (BRASIL,2002).

Reservatórios de maior capacidade volumétrica, abertos ou vedados de forma inadequada, utilizados pela população para fins recreativos ou de abastecimento (cacimbas, caixas-d'água, tanques de cimento e piscinas), mostraram-se estatisticamente associados à ocorrência de casos graves de dengue nos domicílios. Em virtude do maior potencial de produção para as formas adultas do Aedes aegypti, especula-se que a presença desses reservatórios tenha representado aumento na densidade de mosquitos adultos e consequentemente da exposição a picadas (SANTOS, 2012).

A população não está ciente dos problemas ocasionados pelo mau acondicionamento dos resíduos, o qual proporciona um desequilíbrio ambiental e de saúde pública. É fundamental instituir mecanismos de educação ambiental voltado para o melhor acondicionamento dos resíduos e buscar estratégias que propiciem a participação efetiva da população na consolidação de ações que visem o combate à proliferação do mosquito da dengue e por consequência promovam melhoria na saúde e no meio ambiente (OLIVEIRA,2012).

Ao avaliar as práticas de vigilância em um município do estado da Bahia verificou-se que as ações mais implantadas quanto ao programa de controle da dengue municipal foram: o controle químico do vetor e as ações de educação em saúde. Quanto as ações relacionadas ao trabalho intersetorial encontram-se parcialmente implantadas ou não implantadas a exemplo das ações educativas na rede de ensino e a execução de ações de manejo ambiental (drenagem, lixo, esgoto e água). Em relação ao saneamento ambiental não foram referidos projetos de melhoria de fornecimento e armazenagem adequada de água em áreas prioritárias (SILVA; SILVA, 2013). 
A atividade executada para redução dos criadouros do mosquito consistia na distribuição de tampas e capas para reservatórios disponibilizados pelo governo federal. Em nível local, não elaboraram projetos com o propósito de melhorar a frequência do fornecimento de água nas áreas prioritárias conforme estudo epidemiológico e entomológico. Outrossim, os projetos de mutirão de limpeza apesar de sistemáticos não contavam com a adesão da comunidade. Quanto as medidas para estimular o destino adequado do lixo com a implantação de aterro sanitário, na zona rural, grande parte do lixo coletado nas moradias era despejado em "lixão (SILVA; SILVA,2013).

\section{Considerações finais}

As ações de controle ao mosquito Aedes aegypti, se estabelece como uma linha complexa de identificação e resolução de problemas exigindo dos profissionais de saúde, em particular dos ACS e ACE um trabalho integrado, multiprofissional, com objetivos comuns bem definidos buscando a assistência à saúde integral e de qualidade da população.

A realização do estudo permitiu identificar que as ações de enfrentamento ao Aedes aegypti extrapolam o setor saúde sendo indispensável fomentar políticas intersetoriais, ampliar a educação permanente dos profissionais e estimular a participação ativa da sociedade.

Há necessidade da elaboração, implantação e implementação de políticas públicas articuladas, nas três esferas de governo e que considerem medidas eficazes de saneamento básico, com coleta regular de lixo, para reversão do quadro atual das arboviroses, promovendo o controle do vetor e a prevenção de doenças, complicações e óbitos no país.

A integração das ações desenvolvidas pelos setores da Atenção Básica à Saúde e da Vigilância em Saúde/ Saúde Ambiental no controle ao mosquito Aedes aegypti mostrou-se eficaz para o ACS quando desempenha as atribuições do ACE e para este quando é incorporado as rotinas das Equipes de Saúde da Família em consonância com o que é estabelecido pelo PNCD, 2002. 
Com o intuito de potencializar essa cooperação e coordenação entre esses profissionais é fundamental investir em um espaço de diálogo com permanente troca de informações e experiências. Dessa forma, se potencializa o processo de trabalho estabelecendo um mecanismo que fortaleça a integração de indivíduos e desenvolvam consciência de grupo.

O processo de comunicação é mais amplo que a simples transmissão vertical de informações. Desta forma, campanhas veiculadas nas mídias sociais, cartazes/folders devem ser construídos em consonância com a realidade local contando com a participação da comunidade em todas as etapas. Outrossim, atividades de prevenção e promoção a saúde no combate e/ou controle ao mosquito devem ser planejadas e executadas em conjunto com as pessoas valorizando suas crenças, atitudes e comportamentos e promovendo, assim, uma educação em saúde inclusiva e dialógica.

Se faz necessário o envolvimento governamental tripartite no enfrentamento desse vetor e com a participação efetiva dos diversos segmentos da sociedade, contemplando uma linha de financiamento que abarque ações integradas e intersetoriais, de saneamento básico, controle do vetor, vigilância em saúde, educação em saúde, comunicação e mobilização social.

Em virtude da escassez de artigos científicos que abordem sobre a atuação integrada entre os ACS e ACE no combate ao vetor Aedes aegypti sugere-se que novas pesquisas sejam realizadas buscando demonstrar estratégias de atuação e compartilhamento de experiências entre os mais diversos municípios do País.

Outro ponto que merece destaque é o estímulo de recursos materiais e financeiros para o desenvolvimento de pesquisas estratégicas no controle ao mosquito Aedes aegypti no âmbito municipal, com vistas a nortear ações locais mais efetivas a esse problema.

\section{Referências}

BRAGA, I.A.; VALLE, D. Aedes aegypti: histórico do controle no Brasil. Epidemiologia e Serviços de Saúde, v.16, n.2, p.113-118, 2007. Disponível em:< https://www.researchgate net/publication/277125759 Aedes Aegypti historico do controle noBrasil >. Acesso em: 06 de fevereiro de 2016 
BRASIL, Ministério da Saúde (MS). Secretaria de Vigilância em Saúde. Departamento de Vigilância Epidemiológica. Programa Nacional de Controle da Dengue, Brasília: Fundação Nacional de Saúde; 2002.

.Portaria $\mathrm{GM} \mathrm{n}{ }^{\circ}-2122$, de 18 de dezembro de 2015. Altera o Anexo I da Portaria $\mathrm{n}^{\circ}$ 2.488/GM/MS, de 21 de outubro de 2011, para reforçar as ações voltadas ao controle e redução dos riscos em saúde pelas Equipes de Atenção Básica. Diário Oficial da República Federativa do Brasil, seção 1, p.80-81.

CAZOLA, L. H. de O., TAMAKI, E. M.; PONTES, E. R. J. Incorporação do controle da dengue pelo agente comunitário de saúde. Revista Brasileira de Enfermagem, Brasília, v.67, nº 4, jul. /ago.,2014.Disponível em:<http:// .scielo.br/pdf/reben/v67n4/0034-7167-reben-67-04-0637.pdf. Acesso em: 31 de janeiro de 2016.

CHAVES, et al. Dengue, Chikungunya e Zika: a nova realidade brasileira. Revista digital, São Paulo, n 137 , p.132-145.Disponível em: < http://www.scielo.br/pdf/reben/ v67n4/ 0034-7167-reben -67-040637.pdf >. Acesso em :31 de janeiro de 2016.

CESARINO, M. B.; DIBO, M. R.; IANNI, A. M. Z.; VICENTINI, M. E.; FERRAZ, A. A.; NETO; F. C. A difícil interface controle de vetores-atenção básica: inserção dos agentes de controle de vetores da dengue junto às equipes de saúde das unidades básicas no município de São José do Rio Preto Revista Saúde Sociedade, São Paulo, v.23, n³, jul./set.,2014. Disponível em:< http://www.scielo.br/scielo.php? script=sci_arttext\&pid=S0104- 12902014000301 018>. Acesso em: 31 de janeiro de 2016.

GOMES, K. W. L. et al. Organização do processo de trabalho no manejo da dengue em uma capital do Nordeste. Saúde em Debate, Rio de Janeiro, v. 39, n.105, p. 561-569, 2015. Disponível em: <http: //www.scielo.br/ pdf/sdeb/v39 n105 0103-1104-sdeb-39-105-005 61. pdf >. Acesso em: 31 de janeiro de 2016.

LIBANIO, K. R.; FAVORETO, C. A. O.; PINHEIRO, R. Análise da integração de Vigilância Ambiental no controle da dengue com a Estratégia Saúde da Família: impacto nos saberes e práticas doa agentes comunitários de saúde. Revista de Saúde Coletiva, Rio de Janeiro, v.24, n ${ }^{\circ}$, jan. /mar. 2014.Disponível em: $<\quad$ http://www.scielo.br/scielo.php?pid $\quad=S 01037 \quad 33120$ $14000100147 \&$ script=sci_abstract\&tlng=p >. Acesso em: 10 de fevereiro de 2016.

LIMA, E. C.; VILASBOAS, A. L. de Q. Implantação das ações de intersetorialidade de mobilização social para o controle da dengue na Bahia. Cadernos de Saúde Pública. Rio de Janeiro, v.27, n ${ }^{\circ} 8$, agosto, 2011. Disponível em:<http:// www.scielo.br/ pdf/ csp/ v27n8/06.pdf >. Acesso em: $31 \mathrm{de}$ janeiro de 2016.

OLIVEIRA, G. L. A. Prevenção e controle da dengue no município de Sabará/MG: análise de materiais educativos impressos e das representações sociais de agentes de controle de endemias. Tese de Mestrado. Belo Horizonte.2012.Disponível em:< http://arca.icict.fiocruz.br/ bitstream/ icict/5498/1/Dissertacao_Giselle\%20Lopes\%20Armindo\%20de\%20Oliveira.pdf $>$. Acesso em: $10 \mathrm{de}$ fevereiro 2016.

PAIVA, S. A. Dengue versus lixo uma problemática no Jardim Nova Esperança. III Congresso Brasileiro de Gestão Ambiental. Goiânia, 2012. Acesso em: 30 de janeiro de 2016. 
PERES, A. C. Aedes: ampliando o foco. Comunicação e Saúde Revista Radis. Escola Nacional de Saúde Pública.-ENSP Fiocruz, n.161, fev., 2016.

PESSOA, J. P. de M. et al. Controle da dengue: os consensos produzidos por Agentes de Combate às Endemias e Agentes Comunitários de Saúde sobre as ações integradas. Ciências Saúde Coletiva, Rio de Janeiro, v. 21, n8, p. 2329-2338. Disponível em : <http://www. scielo.br/scielo.php?script=sci arttext\&pid=S1413-81232016000802329 >. Acesso em: 11 de agosto de 2016.

REIS, C. B.; ANDRADE, S. M. de O.; CUNHA, R. V. Responsabilização do outro: discursos de enfermeiros da Estratégia Saúde da Família sobre ocorrência de dengue. Revista Brasileira de Enfermagem, Brasília, v.66, $\mathrm{n}^{\circ} 01, \mathrm{jan} / \mathrm{fev}, 2013$. Disponível em: $<\mathrm{http}: / / \mathrm{www} . \quad$ scielo.br/ scielo.php?script=sci_arttext\&pid=S003471672013000100011>. Acesso em: 11 de fevereiro de 2016.

SANTOS, G. B. G. Fatores associados à ocorrência de casos graves de dengue: análise dos anos epidêmicos de 2007-2008 no Rio de Janeiro. Escola Nacional de Saúde Pública Sergio Arouca ENSP. Tese de Doutorado. Disponível em: < http:// bases. bireme.br/cgibin/wxislind.exe/iah/online/?IsisScript=iah/iah.xis\&src=google\&base=LILACS\&lang=p \&nextAction=lnk\&exprSearch=688809\&indexSearch=ID >.Acesso em: 10 de fevereiro de 2016.

SANTOS, S. L.; CABRAL, A. C. dos S. P.; AUGUSTO, L. G.S. Conhecimento, atitude e prática sobre dengue, seu vetor e ações de controle em uma comunidade urbana do Nordeste. Ciência e Saúde Coletiva, Rio de Janeiro, v.16, n.1, jan., 2011. Disponível em: $<$ http://www.scielo.br/ scielo.php?scrip t=sci arttext \&pid= S1413-812 32011000 700066. >. Acesso em: 11 de fevereiro de 2016.

SANTOS, S. L. et al. Percepção sobre o controle da dengue: uma análise a partir do discurso coletivo. Revista Movimentos Sociais e Dinâmicas Espaciais, Recife, v. 03, n. 02, 2015. Disponível em: < http://www.revista.ufpe.br/ revistamseu/index.php/revista/article/ view/130 >. Acesso em: 02 de maio de 2016.

SILVA, L. B.; SOARES, S. M.: FERNANDES, T. de O.; AQUINO, A. L. Comunicação sazonal sobre a dengue em grupos socioeducativos na atenção primária à saúde. Revista de Saúde Pública, São Paulo, v. 45, n.06, p.01-08, 2011.Disponível em: 〈http: //www. scielo. br/scielo.php?pid=S003489102011000600019\&script=sci_arttext $>$. Acesso em: 10de fevereiro de 2016.

SILVA, G. A. P.; SILVA, L. M. V. Organização das práticas de vigilância em um sistema local. Revista Bahiana de Saúde Pública.v.37.n¹, p.57-73, jan/mar, 2013.Disponível em: <http://files.bvs.br/upload/s/01000233/2013/v37n1/a3820.pdf>. Acesso em: 31 de janeiro de 016.

\section{Como citar este artigo (Formato ABNT):}

MESQUITA, Fabíola O. S.; PARENTE, Alaine S.; COELHO, Glória Maria P. Agentes comunitários de saúde e agentes de combate a endemias: desafios para controle do Aedes aegypti. Id on Line Revista Multidisciplinar e de Psicologia, Julho de 2017, vol.11, n.36, p.64-77. ISSN: 1981-1179.

Recebido: 11.06.2017

Aceito: 15.06 .2017 